\title{
The Antitumor Effect of Single-domain Antibodies Directed Towards Membrane-associated Catalase and Superoxide Dismutase
}

\author{
GEORG BAUER $^{1}$ and MANFRED MOTZ ${ }^{2}$ \\ ${ }^{1}$ Institute of Virology, University Medical Center Freiburg, Medical Faculty, \\ University of Freiburg, Freiburg, Germany; \\ ${ }^{2}$ Mikrogen-molekularbiologische Entwicklungs-GmbH, Neuried, Germany
}

\begin{abstract}
Neutralizing single-domain antibodies directed towards catalase or superoxide dismutase (SOD) caused efficient reactivation of intercellular reactive oxygen species/reactive nitrogen species (ROS/RNS)-dependent apoptosis-inducing signaling specifically in human tumor cells. Single-domain antibodies targeted tumor cell-specific membrane-associated SOD and catalase, but not the corresponding intracellular enzymes. They were shown to be about 200-fold more effective than corresponding classical recombinant antigen-binding fragments and more than four $\log$ steps more efficient than monoclonal antibodies. Combined addition of single-domain antibodies against catalase and SOD caused a remarkable synergistic effect. Proof-of-concept experiments in immunocompromised mice using human tumor xenografts and single-domain antibodies directed towards SOD showed an inhibition of tumor growth. Neutralizing single-domain antibodies directed to catalase and SOD also caused a very strong synergistic effect with the established chemotherapeutic agent taxol, indicating an overlap of signaling pathways. This effect might also be useful in order to avoid unwanted side-effects and to drastically lower the costs for taxol-based therapy.
\end{abstract}

The transition from the non-transformed to the transformed state of cells is characterized by sustained generation of extracellular superoxide anions by membrane-associated

This article is freely accessible online.

Correspondence to: Georg Bauer, Institut für Virologie, Department für Medizinische Mikrobiologie und Hygiene, Hermann-Herder Strasse 11, D-79104 Freiburg, Germany. E-mail: georg.bauer@uniklinikfreiburg.de

Key Words: Single-domain antibody, catalase, SOD, ROS, RNS apoptosis.
NADPH oxidase-1 (NOX1) (1-4). Dismutation of superoxide anions results in the generation of $\mathrm{H}_{2} \mathrm{O}_{2}$ that enters the cells through aquaporins $(5,6)$ and drives their proliferation and contributes to the maintenance of the transformed state (2).

NOX1-derived superoxide anions drive two intercellular reactive oxygen species/reactive nitrogen species (ROS/RNS)-dependent apoptosis-inducing signaling pathways that selectively eliminate transformed cells through induction of apoptosis (4). These are the $\mathrm{HOCl}$ signaling pathway $(4,7,8)$ and the $\mathrm{NO}$ /peroxynitrite pathway $(4,7,9)$.

Bona fide tumor cells, representing a late stage of tumor progression, are characterized by their resistance towards exogenous $\mathrm{H}_{2} \mathrm{O}_{2}(10,11)$. This is mediated by membraneassociated catalase (12-14) which tightly controls and prevents $\mathrm{HOCl}$ signaling through decomposition of $\mathrm{H}_{2} \mathrm{O}_{2}$ and $\mathrm{NO}$ /peroxynitrite signaling through oxidation of $\mathrm{NO}$ and decomposition of peroxynitrite $(4,13)$. Compared to transformed cells, bona fide tumor cells exhibit stronger activity of NOX1 (Bauer, unpublished data), have an increased number of aquaporins that facilitate uptake of $\mathrm{H}_{2} \mathrm{O}_{2}$ (15) and seem to have a reduced concentration of intracellular catalase (14). These phenotypic features seem to allow for optimal stimulation of proliferation through intracellular $\mathrm{H}_{2} \mathrm{O}_{2}$, while the cells are protected against extracellular ROS/RNS-dependent signaling. Tumor cells also need to express sufficient membrane-associated superoxide dismutase (SOD) $(3,16)$ to lower the concentration of free superoxide anions below their inhibitory level for catalase (17-19).

The combination of the tumor cell-specific membraneassociated enzymes NOX1, SOD and catalase allows for the selective induction of ROS/RNS-mediated apoptosis in tumor cells through direct inhibition or inactivation of catalase $(4,12,13)$. Alternatively, as deduced from the interaction between NOX1, SOD and catalase, the inhibition of SOD should cause indirect inhibition of catalase through free superoxide anions, followed by ROS/RNS-dependent apoptosis-inducing signaling. 
We endeavored to clarify whether targeting the protective system of tumor cells against ROS/RNS signaling with neutralizing antibodies directed towards catalase or SOD would establish a selective apoptotic response through reactivation of intercellular ROS/RNS signaling. Therefore, an approach with antigen-binding fragments of antibodies seemed to be the most appropriate way to answer this question, as it excludes immunological processes controlled by the Fc entity of the antibody and thus allows focus on the effects induced by the inhibited target structure.

Single-domain antibodies, consisting of the variable chain of a heavy chain-only antibody (VHH) of camelids, exhibit surprising properties such as high affinity, the ability to inhibit enzymes, high potential to penetrate into tumor tissue, high stability and, due to their small size, seem to be nonimmunogenic $(20,21)$. For this reason, we endeavoured to generate and characterize single-domain antibodies that reactivate intercellular ROS/RNS signaling specifically in tumor cells through inhibition of either membrane-associated catalase or SOD.

\section{Materials and Methods}

Materials. The NADPH oxidase 1 (NOX1) inhibitor 4-(2aminoethyl)benzenesulfonyl fluoride (AEBSF), neutralizing monoclonal antibody directed against human catalase (anti-CAT) (mouse, IgG1, clone CAT-505), neutralizing monoclonal antibody directed against human SOD1 (anti-SOD) (mouse, IgG1, clone SDG6), control antibody directed against laminin, the $\mathrm{HOCl}$ scavenger taurine, $\mathrm{H}_{2} \mathrm{O}_{2}$-generating glucose oxidase (GOX) from Aspergillus niger, the singlet oxygen scavenger histidine and taxol (paclitaxel from Taxus brevifolia) were obtained from Sigma Aldrich (Schnelldorf, Germany).

The peroxynitrite decomposition catalyst 5-, 10-, 15-, 20-tetrakis(4sulfonatophenyl)porphyrinato iron(III) chloride (FeTPPS) was obtained from Calbiochem/Merck Biosciences GmbH, Schwalbach, Germany.

Recombinant neutralizing human antigen-binding fragments (Fab) directed towards human catalase (\#AbD15562), Format FabV5Sx2, and recombinant neutralizing human Fab fragments directed towards human SOD1 (\#AbD15660) Format Fab-V5Sx2 were purchased from AbDSerotec (Munich). The Fab fragments had been generated from a HuCAL ${ }^{\circledR}$ library according to the procedures described in European patent 859841 and US patent 6,300,064.

Recombinant camelid single-domain antibodies (VHH) directed against human catalase and SOD were generated and produced by ChromoTek GmbH, Martinsried (Germany)/www.chromotek.com. Their amino acid sequence and the nucleotide sequence of the corresponding clones were determined by ChromoTek.

Alpacas were immunized with human catalase (Sigma Aldrich) or human SOD1 (Sigma Aldrich) under the control of a veterinarian. RNA was isolated from B-cells of the immunized animals, transcribed with RNA-dependent DNA polymerase, cloned in Escherichia coli. Isolation of positive clones was based on phage display technology. Clones that coded for single-domain VHHs directed towards catalase or SOD were identified by testing their supernatants on appropriate enzyme-linked sorbent assays. Isolated clones were purified and their DNA was sequenced according to standard techniques. Production of milligram amounts of VHHs followed standard procedures.

The single-domain VHHs aCATcb0972 and aCATcb0974 bind to human catalase and neutralize the enzyme activity, whereas VHHs aCATcb0973 and aCATcb0975 bind to catalase but do not neutralize it. ASODcb0989 binds to human SOD1 and neutralizes the enzyme, whereas aSODcb0987 and aSODcb0991 bind to SOD1 but do not neutralize it. ACATcb0972 and aSODcb0989 were used for the generation of hybrid VHHs by connecting the two VHHS by a linker.

Media for cell culture. Cells were either kept in Eagle`s minimal essential medium (EMEM) containing 5\% FBS (Biochrom, Berlin, Germany), or in RPMI-1640 medium containing 10\% FBS, as indicated for the respective cell lines. FBS was heated for 30 minutes at $56^{\circ} \mathrm{C}$ prior to use. Both media were supplemented with penicillin (40 U/ml), streptomycin $(50 \mu \mathrm{g} / \mathrm{ml})$, neomycin $(10 \mu \mathrm{g} / \mathrm{ml})$, moronal $(10 \mathrm{U} / \mathrm{ml})$ and glutamine $(280 \mu \mathrm{g} / \mathrm{ml})$. All supplements were obtained from Biochrom, Berlin, Germany. Cell culture was performed in plastic tissue culture flasks. Cells were passaged once or twice weekly.

Cells. Nontransformed rat fibroblasts $208 \mathrm{~F}$ and their derivative transformed through constitutive expression of v-src (208Fsrc3) were a generous gift from Dr C. Sers and Dr R. Schäfer, Berlin, Germany and were cultured in EMEM with 5\% FBS and supplemented as indicated above.

The gastric carcinoma cell line MKN-45 was purchased from the Deutsche Sammlung für Mikroorganismen und Zellkulturen, Braunschweig, Germany. Cells were grown in suspension, with some cells attaching to the plastic culture dish, in RPMI-1640, with $10 \%$ serum and supplements as described above. Care was taken to avoid cell densities below $300,000 / \mathrm{ml}$ and above $106 / \mathrm{ml}$.

The human neuroblastoma cell line SHEP was obtained from Dr. J. Roessler, Department of Pediatrics and Adolescent Medicine, University Medical Centre Freiburg, Germany and was cultured in EMEM with 5\% FBS and supplemented as indicated above.

Non-malignant diploid human fibroblasts Alpha-1 had been obtained from the Diagnostic Unit of our Institute and had been prepared from the foreskin of a healthy volunteer.

Small interfering ribonucleic acid (siRNA)-mediated knockdown of NOX1, inducible NO synthase-2 (iNOS2), FAS receptor (FASR; a member of the tumor necrosis factor receptor family), caspase-8, and caspase-9. Control siRNA ( $\mathrm{siCo}$ ), siRNA against human NOX1 (siNOX1), human iNOS2 (siiNOS2), human FASR (siFASR), human caspase-8 (siCASP-8), human caspase-9 (siCASP-9) were obtained from Qiagen (Hilden, Germany) with the following sequences: siCo: sense: r(UUCUCCGAACGUGUCACGU)dTdT, antisense: ACGUGACACGUUCGGAGAA)dTdT; custom-made siNOX1: sense: r(GACAAAUACUACUACACAA)dTdT, antisense: r(UUGUGUAGUAGUAUUUGUC)dGdG; custom-made siRNA towards dual oxidase-1 (siDUOX1): sense: r(AGU CUA ACA CCA CAA CUA A)dTdT, antisense: r(UUA GUU GUG GUG UUA GAC U)dGdG; custom-made siiNOS2: sense r(GGG CCG UGC AAA CCU UCA A)dTdT; antisense: r(UUG AAG GUU UGC ACG GCC C)dAd; siFASR (Hs_FAS_7_HP Validated siRNA, Cat. Nr. SI02654463, target sequence: AAG GAG TAC ACA GAC AAA GCC), no information on the sequence of siFASR was available from the supplier; siCASP-8 (Hs_CASP8_11_HP Validated siRNA, Cat- Nr. SI02661946, target of sequence: AAG AGT CTG TGC 
CCA AAT CAA), no information on the sequence of siCASP-8 was available from the supplier; siCASP-9 (Hs_CASP9_7_HP Validated siRNA, Cat. Nr. SI02654610, target of sequence: CAG TGA CAT CTT TGT GTC CTA), no information on the sequence of siCASP9 was available from the supplier.

siRNAs were dissolved in suspension buffer supplied by Qiagen at a concentration of $20 \mu \mathrm{M}$. Suspensions were heated at $90^{\circ} \mathrm{C}$ for 1 minute, followed by incubation at $37^{\circ} \mathrm{C}$ for 60 minutes. Aliquots were stored at $-20^{\circ} \mathrm{C}$.

Before transfection, $88 \mu \mathrm{l}$ of medium without serum and without antibiotics were mixed with $12 \mu \mathrm{l}$ Hyperfect solution (Qiagen) and $1.2 \mu \mathrm{l}$ specific siRNA or control siRNA. The mixture was treated by a Vortex mixer for a few seconds and then allowed to sit for 10 minutes. It was then gently and slowly added to $300,000 \mathrm{MKN}-45$ cells in $1 \mathrm{ml}$ RPMI-1640 medium containing 10\% FBS and antibiotics (12-well plates) Thus the concentration of siRNA was 24 $\mathrm{nM}$. The cells were incubated at $37^{\circ} \mathrm{C}$ in $5 \% \mathrm{CO}_{2}$ for 24 hours. Transfected cells were centrifuged and resuspended in fresh medium at the required density before use.

Determination of the efficiency of siRNA-mediated knockdown. The siRNA transfection system as described above had been optimized to allow a reproducible transfection efficiency of more than $95 \%$ of the cells and to avoid toxic effects (Bauer, unpublished data).

The efficiency of specific knockdown of FASR, caspase- 8 and caspase- 9 mRNA by siFASR, siCASP-8, siCASP- 9 had been experimentally determined by the supplier, using real time polymerase chain reaction and was found to be more than $90 \%$ for $5 \mathrm{nM}$ siRNA.

The efficiency of knockdown by siNOX and siiNOS was based on functional assays.

Knockdown of NOX1 was found to be $94 \%$ following the protocol described recently (22). After knockdown of iNOS, NOdependent effects were undetectable.

Autocrine ROS/RNS-mediated apoptosis induction. When seeded at appropriate density as well as cell number, tumor cells (in the presence of an inhibitor or inactivator of their protective catalase) establish apoptosis-inducing intercellular ROS/RNS signaling. Assays were performed in 96-well plates with $100 \mu \mathrm{l}$ of complete medium per well and contained either $12,500 \mathrm{MKN}-45$ cells or 10,000 SHEP cells per well. Cells received the respective antibodies at appropriate concentrations. Inhibitors were added at the following final concentrations: AEBSF $100 \mu \mathrm{M}$, taurine $50 \mathrm{mM}$, FeTPPS $20 \mu \mathrm{M}$, or histidine $2 \mathrm{mM}$. All assays were performed in duplicate. In one experiment, MKN-45 cells received increasing concentrations of taxol plus single-domain antibodies. The plates were incubated at $37^{\circ} \mathrm{C}$ in an atmosphere of $5 \% \mathrm{CO}_{2}$. The percentages of apoptotic cells were then determined after 3.5-4 hours for MKN-45 cells and 5-6 hours for SHEP cells as described below.

Challenging of cells with $\mathrm{H}_{2} \mathrm{O}_{2}$-generating $\mathrm{GOX}$ for the determination of catalase activity. Cells were seeded at a 6,000 cells per $100 \mu \mathrm{l}$. After the cells had attached, they were treated with single-domain antibodies for 20 minutes at $37^{\circ} \mathrm{C}$. Then GOX was added $(0 ; 0.015-20 \mathrm{mU} / \mathrm{ml}$ in twofold dilution steps) in duplicate assays. The percentages of apoptotic cells were determined after 1.5 hours as described below. Apoptosis induction in this experiment is based on $\mathrm{H}_{2} \mathrm{O}_{2}$-dependent apoptosis induction $(14,23)$ and not on intercellular ROS signaling. The degree of sensitivity reflects the lack of protective catalase (14).
Determination of the percentage of apoptotic cells. The percentage of apoptotic cells was determined by inverted phase-contrast microscopy based on the classical criteria for apoptosis, i.e. nuclear condensation or fragmentation and membrane blebbing (24-27). At least $2 \times 200$ cells were scored for each point of measurement in duplicate assays.

In vivo experiments. In vivo experiments for the evaluation of antitumor effects of neutralizing single-domain antibodies were performed by Oncotest GmbH, Freiburg (Germany).

All experiments were approved by the local authorities, and were conducted according to all applicable international, national and local laws and guidelines. Only animals with unobjectionable health were selected to enter testing procedures. Animals were routinely monitored twice daily on working days and daily on Saturdays and Sundays.

Female athymic nude mouse NMRI $n u / n u$ were used. These are immunodeficient, thus enabling the xenotransplantation and growth of human tumors. Animals were arbitrarily numbered at the beginning of the experiment using radiofrequency identification transponders. The animals were housed in individually ventilated cages (TECNIPLAST Sealsafe ${ }^{\mathrm{TM}}$-IVC-System; TECNIPLAST, Hohenpeissenberg, Germany) and were kept under a 14:10 light:dark artificial light cycle.

The patient-derived colon carcinoma xenograft CXF 975 used by Oncotest was derived from surgical specimens from cancer patients. Tumor fragments were obtained from xenografts in serial passage in nude mice. After removal from donor mice, tumors were cut into fragments (4-5 mm diameter) and placed in PBS until subcutaneous implantation. Mice under isofluorane anesthesia received unilateral, subcutaneous tumor implants in the flank.

Only mice carrying a tumor of appropriate size $\left(50 \mathrm{~mm}^{3}\right.$ to $250 \mathrm{~mm}^{3}$ ) were considered for randomization. Mice were randomized when the required number of mice qualified for randomization. The day of randomization was designated as day 0 . At randomization, tumor-bearing animals were stratified into different groups according to tumor volume. All antibodies and the vehicle were given intravenously twice weekly $(10 \mathrm{ml} / \mathrm{kg})$. Antitumor activity (tumor growth inhibition) and tolerability were assessed using the vehicle control group as a reference. The treatment periods were 21 to 25 days followed by an observation period that lasted until day 32. Tumor and blood samples were collected for further analysis. The absolute tumor volumes (ATV) were determined by two-dimensional measurement with a caliper on the day of randomization and then twice weekly (i.e. on the same days on which mice were weighed). Tumor volumes were calculated according to the formula: $\left(a \times b^{2}\right) \times 0.5$ where $a$ represents the largest and $b$ the perpendicular tumor diameter.

Relative volumes of individual tumors (individual RTVs) for day $x$ were calculated by dividing the individual tumor volume on day $x\left(\mathrm{~T}_{x}\right)$ by the individual volume of the same tumor on day $0\left(\mathrm{~T}_{0}\right)$ multiplied by $100 \%$.

Statistical analysis. Assays were performed in duplicate, unless otherwise stated. The empirical standard deviation was calculated and is shown in the figures. Absence of standard deviation bars for certain points indicates that the standard deviation was too small to be reported by the graphic program. Empirical standard deviations were calculated merely to determine how close the results were obtained in parallel assays within the same experiment and not with 
the intention of statistical analysis of variance. The Yates continuity corrected chi-square test was used for the statistical determination of significances.

For clarity, the figures use a logarithmic scale annotated with the actual concentration applied.

\section{Results}

Single-domain VHHs that bind to human catalase $(n=4)$ or human SOD1 $(n=3)$ were generated and tested for their potential to cause inhibition of membrane-associated catalase, followed by reactivation of intercellular apoptosisinducing signaling. As shown in Figure 1A and C, two catalase-binding (aCATcb0972 and aCATcb0974) and one SOD-binding (aSODcb0989) single-domain VHH caused apoptosis induction, dependent on their concentration. In contrast, two of the catalase-binding (aCATcb0973, aCATcb0975) and two of the SOD-binding (aSODcb087 and aSODcb091) single-domain VHHs did not cause apoptosis induction in MKN-45 human gastric carcinoma cells, indicating that they only bound to but did not inhibit the targeted enzymes. In the case of aCATcb0972 and aCATcb0974, an initial optimum curve of apoptosis induction (optimum at $1.2 \mathrm{pg} / \mathrm{ml}$ ) was followed by a second rise in apoptosis induction at higher concentrations (Figure 1A). Apoptosis induction by aSODcb0989 was characterized by an optimum curve in the same concentration range as seen for neutralizing single-domain antibodies directed towards catalase, but without a strong secondary rise. Classical recombinant Fabs directed towards human catalase or SOD required about 250 -fold higher concentrations than the corresponding single-domain VHHs in order to obtain the same apoptosis-inducing effect (Figure 1B and D).

Apoptosis induction by all concentrations of aCATcb0972 was inhibited by the NOX1 inhibitor AEBSF, indicating an underlying superoxide anion-dependent process (Figure 2A). Apoptosis induction at very low concentrations of aCATcb0972 was dependent on NO/peroxynitrite signaling and independent of $\mathrm{HOCl}$ signaling, as it was inhibited by the peroxynitrite decomposition catalyst FeTPPS (Figure 2B), but not by the $\mathrm{HOCl}$ scavenger taurine (Figure 2A). At concentrations up to $6.2 \mathrm{pg} / \mathrm{ml}$, apoptosis induction seemed to completely depend on $\mathrm{HOCl}$ signaling, as seen by the complete inhibition by taurine and the lack of inhibition by FeTPPS. At higher concentrations, neither $\mathrm{HOCl}$ nor $\mathrm{NO}$ /peroxynitrite signaling seemed to be responsible, although the process was driven by superoxide anions. Interestingly, the singlet oxygen scavenger histidine, which had no inhibitory effect on apoptosis induction, mediated the supraoptimal decline of apoptosis induction beyond $0.75 \mathrm{pg} / \mathrm{ml}$. FeTPPS had an analogous effect at this concentration range.

In contrast, apoptosis induction by aSODcb0989 seemed to be dependent on $\mathrm{NO}$ /peroxynitrite signaling at all concentrations, without an indication for $\mathrm{HOCl}$ signaling, as determined by the inhibition profile (Figure 2C and D). Whereas apoptosis induction by aCAT was strongly dependent on cell density, this effect was much less pronounced for aSOD (data not shown).

For functional determination of specificity and extracellular location of anti-catalase activity and of interspecies crossreactivity, nontransformed (208F) and srconcogene-transformed rat fibroblasts (208Fsrc3) and human MKN-45 gastric carcinoma cells were analyzed with respect to protection from exogenous $\mathrm{H}_{2} \mathrm{O}_{2}$ through membraneassociated catalase. As shown in Figure 3A, when challenged with $\mathrm{H}_{2} \mathrm{O}_{2}$ generated by increasing concentrations of glucose oxidase (GOX), 208F and 208Fsrc3 cells were much more sensitive than tumor cells. However, catalase-binding and neutralizing aCATcb0972 rendered MKN-45 more sensitive than nontransformed cells in a concentration-dependent mode, whereas the binding but not-neutralizing aCATcb0973 had no effect (Figure 3B). Similarly, aCATcb072, but not aCATcb073, increased the sensitivity of 208Fsrc3 cells but not that of nontransformed 208F cells (Figure 3C and D). These findings show that single-domain VHHs specifically target extracellular catalase and confirm that tumor cells have higher cell-surface concentrations of protective catalase than transformed cells, whereas nonmalignant cells have no such membrane-associated catalase. When increasing concentrations of aCATcb0972 were combined with a very low concentration of antiSODcb0989, or increasing concentrations of antiSODcb0989 were combined with a very low concentration of antiCATcb0972, a remarkable synergistic effect on apoptosis induction was seen (Figure 4).

Hybrid molecules that included a catalase- and an SODneutralizing $\mathrm{VHH}$ domain were much more efficient in apoptosis induction than individually applied neutralizing VHHs (Figure 5). The concentration required for optimal apoptosis induction of the two hybrid single-domain antibodies (differing solely in the order of the domains) was more than 200-fold lower than that of individually applied single-domain antibodies directed towards catalase or SOD. This finding therefore demonstrates that the synergistic effect can be bundled in one hybrid molecule.

Apoptosis induction by the aCATaSOD hybrid singledomain antibody was dependent on its concentration and seemed to be solely driven by $\mathrm{NO}$ /peroxynitrite signaling, as it was inhibitied by the NOX1 inhibitor AEBSF and the peroxynitrite decomposition catalyst FeTPPS but not by the HOCl scavenger taurine (Figure 5). Apoptosis induction was independent of singlet oxygen, but scavenging singlet oxygen through application of histidine caused an abrogation of the supraoptimal decline. siRNA-mediated knockdown of NOX1 or $i N O S$ caused complete prevention of apoptosis induction by the hybrid, confirming the role of $\mathrm{NO}$ /peroxynitrite signaling (Figure 6). Inhibition of apoptosis after knockdown of caspase- 9 points to the execution of the mitochondrial 


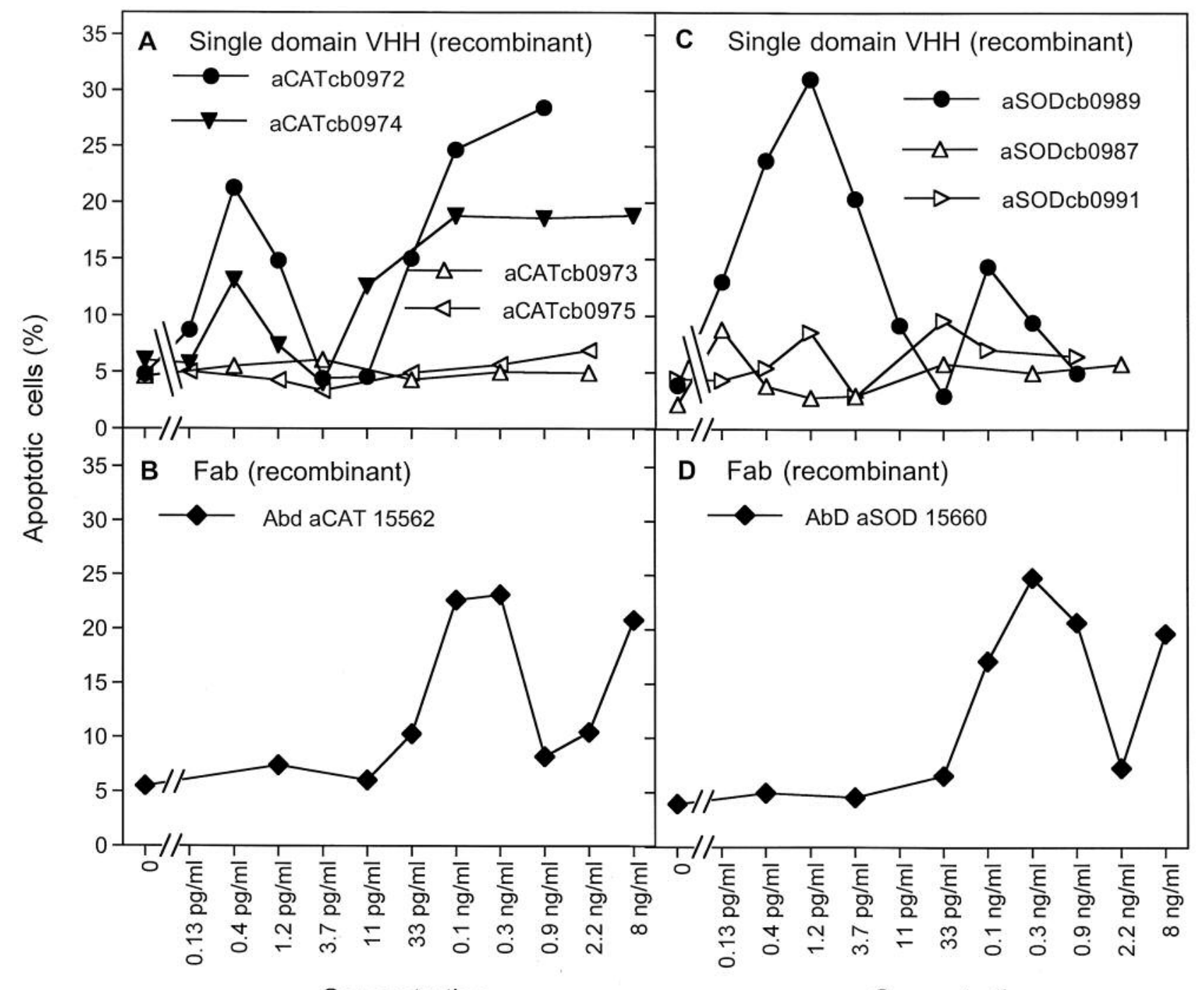

Concentration

Concentration

Figure 1. Apoptosis induction in MKN-45 human gastric carcinoma cells by neutralizing single-domain antibodies and antibody-binding fragments (Fabs) directed towards human catalase $(A, B)$ or superoxide dismutase (SOD) (C, D). A: Catalase-neutralizing aCATcb0974 and aCATcbo072 single-domain antibodies induced apoptosis in MKN-45 cells, whereas non-neutralizing single-domain antibodies that only bind to catalase (aCATcb0973 and aCATcb0975) had no apoptosis-inducing effect. B: Neutralizing classical recombinant Fab fragment AbdaCAT15562 directed towards catalase induced apoptosis in MKN-45 cells. Compared to single-domain antibodies, a 250-fold higher concentration of Fabs were required in order to achieve the same effect. C: SOD-neutralizing aSODcb0989 single-domain antibodies mediate apoptosis induction in MKN-45 cells, whereas non-neutralizing, but SOD-binding aSODcb0987 and aSODcb0991 had no apoptosis-inducing effect. D: The recombinant SOD-neutralizing Fab AbDaSOD15660 caused apoptosis in MKN-45 cells with 300-fold lower efficiency than neutralizing single-domain antibodies. This initial experiment was performed with single determinations and repeated twice (data not shown).

pathway of apoptosis. In contrast, knockdown of either the FASR or of caspase-8 had no inhibitory effect on apoptosis induction by the hybrid single-domain antibody but abrogated the supraoptimal decline of the optimum curve.

The supraoptimal decline is due to excess $\mathrm{H}_{2} \mathrm{O}_{2}$ that interferes with $\mathrm{HOCl}$ signaling in multiple ways. The preventive effect of the singlet-oxygen scavenger histidine and the knockdown of FASR or caspase- 8 indicates that singlet-oxygen generation, enhanced by FASR through caspase- 8 , causes supraoptimal inactivation of catalase.

The neuroblastoma cell line SHEP represents a characteristic example of tumor cell lines that preferentially exhibits $\mathrm{NO} /$ peroxynitrite signaling after inhibition of catalase (13). As shown in Figure 7A, aCATcb0972 and aSODcb0989 


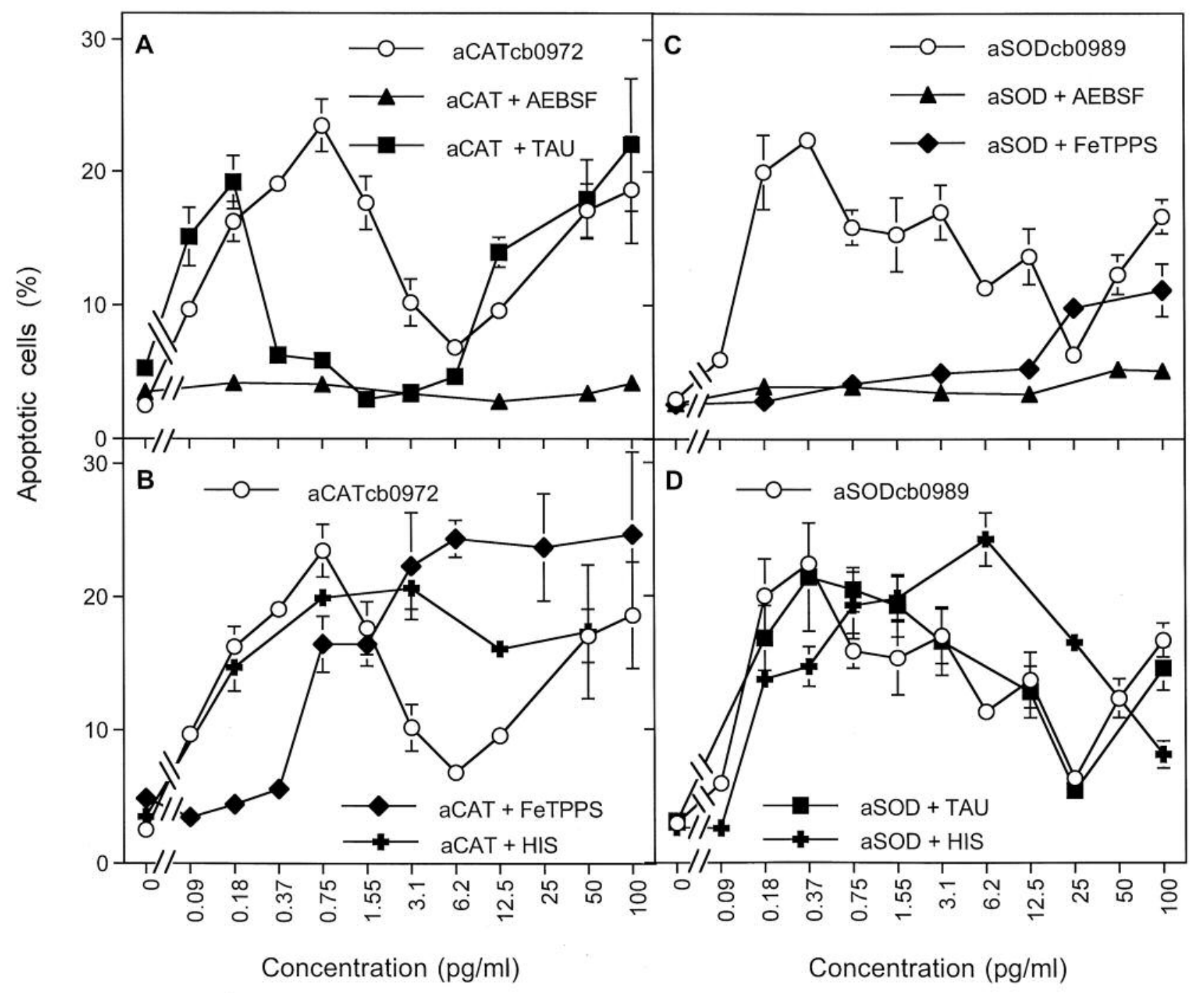

Figure 2. Differential reactivation of reactive oxygen species/reactive nitrogen species signaling by neutralizing single-domain antibodies directed towards catalase $(a C A T)(A, B)$ and superoxide dismutase $(S O D)(a S O D)(C, D)$. Application of the NADPH oxidase-1 inhibitor inhibitor 4-(2aminoethyl)benzenesulfonyl fluoride (AEBSF), the HOCl scavenger taurine (TAU) (A), and the peroxynitrite decomposition catalyst 5-, 10-, 15-, 20-tetrakis(4-sulfonatophenyl)porphyrinato iron(III) chloride (FeTPPS) and the singlet oxygen scavenger histidine (HIS) (B) to MKN-45 cells shows that neutralizing single-domain antibodies directed towards catalase reactivate NO/peroxynitrite signaling at low concentrations, followed by $\mathrm{HOCl}$ signaling at higher concentrations and a superoxide anion-dependent process at very high concentrations, whereas singlet oxygen plays no role. Neutralizing single-domain antibodies directed towards SOD reactivate NO/peroxynitrite signaling exclusively $(C$ and $D)$.

caused apoptosis induction in SHEP cells very efficiently, following a plateau-like curve over the fento- to nanogram per milliliter concentration range. Non-neutralizing aSODcb0991 caused extremely weak apoptosis induction and only at extremely high concentrations. Apoptosis induction by the hybrid single-domain antibody aCATaSOD gave a similar overall picture as induced by single-domain antibodies directed towards individual enzymes, although, as expected, was effective at much lower concentrations. For example, $0.001 \mathrm{fg} / \mathrm{ml}$ of the hybrid molecule caused the same apoptosis- inducing effect on SHEP cells as $1.6 \mathrm{fg} / \mathrm{ml}$ aCATcb0972 or $5 \mathrm{fg} / \mathrm{ml}$ aSODcb0989.

The combination of low concentrations of the neutralizing single-domain antibodies aCATcb0972 or aSODcb0989, but not of the non-neutralizing aSODcb0987, caused a remarkable synergistic effect with the established chemotherapeutic agent taxol when applied to MKN-45 tumor cells (Figure 8). This confirms our recent finding that taxol also targets the protective catalase/SOD system of tumor cells and reactivates intercellular apoptosis-inducing signaling. 
Our findings on apoptosis induction in tumor cells in vitro through application of neutralizing aSODcb0989 were paralleled by a significant effect of aSODcb0989 on a human colon carcinoma xenotransplant in immunecompromised mice (Figure 9). Most of the animals treated with $0.3 \mathrm{mg} / \mathrm{kg}$ aSODcb0989 twice a week showed reduced tumor growth compared to the control group, whereas $0.03 \mathrm{mg} / \mathrm{kg}$ had no significant effect. Further increase of single-domain antibodies to $0.9 \mathrm{mg} / \mathrm{kg}$ caused a very strong effect in three animals, whereas three animals were without effect. This particular finding points to an optimum curve of action in vivo. Application of aCATcb0972 in vivo, using the same xenograft and mode of application, showed an optimum of $0.3 \mathrm{mg} / \mathrm{kg}$ single-domain antibody. However, the overall effect of aCATcb0972 was only about half as strong as that of aSODcb0989 (data not shown). Comparison of the median relative tumor growth at day 25 of treatment showed that aSODcb0989 caused 55\% inhibition of tumor growth, whereas aCATcb0972 only caused $33 \%$ of inhibition.

\section{Discussion}

Our data show that neutralizing single-domain antibodies (VHHs) directed towards membrane-associated catalase or SOD reactivate intercellular ROS/RNS-dependent intercellular apoptosis-inducing signaling specifically of tumor cells with valuable efficiency.

As the $\mathrm{HOCl}$ and the $\mathrm{NO}$ /peroxynitrite pathways are under dominant control by membrane-associated catalase of tumor cells (13), their reactivation by aCATcb0972 and aCATcb0974 is obviously due to efficient neutralization of the enzyme by these VHHs. This conclusion is further supported by the finding that treatment of tumor cells with these VHHs sensitized the tumor cells for a subsequent challenge by $\mathrm{H}_{2} \mathrm{O}_{2}$ (generated by GOX), whereas there was no sensitizing effect on non-malignant cells that lack membrane-associated catalase. Therefore, these findings also demonstrate that intracellular catalase is not affected by the neutralizing VHHs directed towards catalase. The minor sensitizing effect on transformed cells is due to their suboptimal concentrations of catalase (14).

Reactivation of NO/peroxynitrite signaling after inhibition of membrane-associated SOD by the neutralizing aSODcb0989 indicates that the inhibition of SOD must have affected membrane-associated catalase in an indirect way, as $\mathrm{NO}$ /peroxynitrite signaling is under tight control by catalase $(4,13)$. This interaction is explained by the high concentration of free NOX1-derived superoxide anions after inhibition of SOD, followed by superoxide anion-dependent inhibition of catalase (17-19).

The lack of enzymatic dismutation of superoxide anions after inhibition of SOD explains why this approach leads

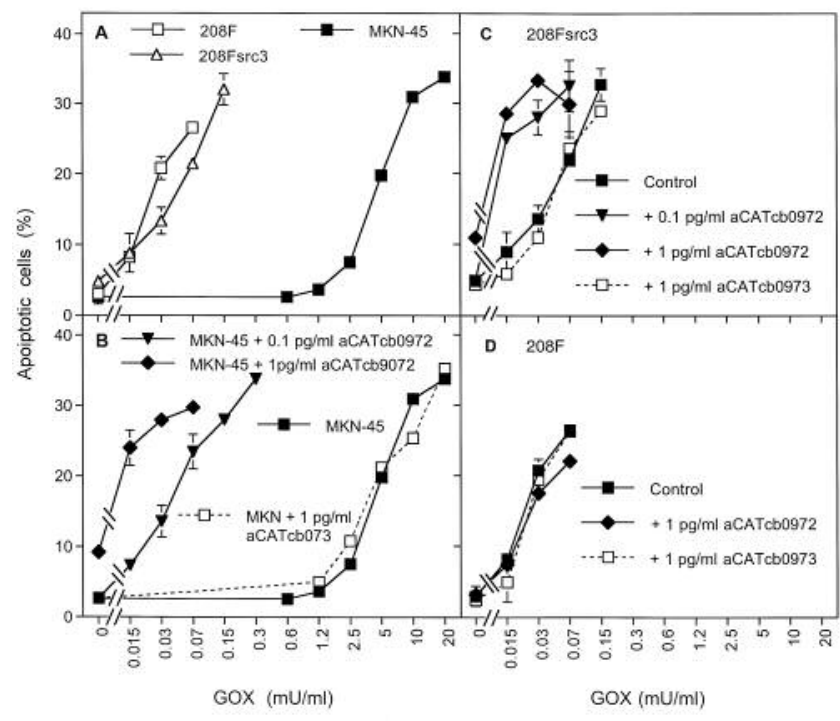

Figure 3. Neutralizing single-domain antibodies directed towards catalase sensitize malignant cells for apoptosis induction by exogenous $\mathrm{H}_{2} \mathrm{O}_{2}$ but have no effect on non-malignant cells. A: MKN-45 gastric carcinoma cells exhibited much higher resistance towards exogenous $\mathrm{H}_{2} \mathrm{O}_{2}$ generated by glucose oxidase (GOX) than non-malignant rat $208 \mathrm{~F}$ and transformed $208 \mathrm{Fsrc} 3$ cells. B: MKN-45 tumor cells were sensitized by neutralizing single-domain antibodies directed towards catalase (aCATcb0972), whereas binding but non-neutralizing singledomain antibodies aCATcb073 had no effect. C: Transformed 208 Fsrc 3 cells were sensitized by neutralizing single-domain antibodies directed towards catalase (aCATcb0972), whereas binding but non-neutralizing single-domain antibodies aCATcb073 had no effect. D: Due to the lack of expression of membrane-associated catalase, non-malignant $208 \mathrm{~F}$ cells were not affected by neutralizing single-domain antibodies directed towards catalase.

exclusively to signaling through the $\mathrm{NO} /$ peroxynitrite pathway, since $\mathrm{H}_{2} \mathrm{O}_{2}$ required for peroxidase-dependent $\mathrm{HOCl}$ synthesis is not generated at a sufficient concentration under these conditions.

Taken together, apoptosis induction in tumor cells by neutralizing single-domain antibodies directed towards catalase or SOD is characterized by high efficiency and selectivity, as it is based on the concerted action of two specific features of tumor cells, namely active NOX1 and the combined action of catalase/SOD on the surface of the tumor cell. This opens the chance for a novel approach for in vivo tumor therapy.

These conclusions are further substantiated by the finding that siRNA-mediated knockdown of NOX1 rendered tumor cells insensitive to apoptosis induction by single-domain antibodies (Figure 6), whereas knockdown of catalase had the same effect as neutralization of catalase by antibodies $(13,14)$. Importantly, single-domain VHHs had no apoptosis inducing effect on normal diploid human fibroblasts (data not shown). 
In vitro, single-domain neutralizing antibodies were more effective at reactivating intercellular ROS/RNS-dependent apoptosis induction in tumor cells than classical recombinant neutralizing Fabs consisting of heavy and light chain variable regions, and even more effective than complete neutralizing antibodies. In order to obtain optimal apoptosis induction in MKN-45 gastric carcinoma cells, $100 \mathrm{ng} / \mathrm{ml}$ monoclonal antibodies directed towards SOD or catalase were required, whereas $0.2 \mathrm{ng} / \mathrm{ml}$ of recombinant Fab (Abd Serotec) directed towards catalase, $0.3 \mathrm{ng} / \mathrm{ml}$ recombinant Fab (Abd Serotec) directed towards SOD, 0.4 $\mathrm{pg} / \mathrm{ml}$ single-domain antibody directed towards catalase and $1.2 \mathrm{pg} / \mathrm{ml}$ single-domain antibody directed towards SOD caused the same effect. When single-domain VHHs directed towards catalase and SOD were combined in a hybrid molecule, only $0.25 \mathrm{fg} / \mathrm{ml}$ of this hybrid molecule were needed for optimal apoptosis induction. The better performance of single-domain antibodies compared to classical Fabs is not explained by the difference in molecular mass. Hypothetically, the intrinsic nature of single-domain antibodies to bind into cavities on their target may establish their strong inhibitory effect on enzymes such as SOD and catalase that have a tunnel-like structure leading to their active center. In addition, it is conceivable that single-domain antibodies bind to their target with a similar reaction rate as conventional Fabs but that binding of single-domain antibodies into cavities leads to a conformational changes of the target that prevents the back reaction of the antibodies and thus causes a dramatic increase in overall affinity.

The data obtained with neutralizing single-domain antibodies in vivo can be taken as a first proof of principle for the antitumor effect of single-domain antibodies that target the protective system of tumor cells. They also indicate that single-domain antibodies directed towards SOD are more effective than those directed towards catalase. One conceivable advantage of VHHs directed towards SOD is the reduction of free $\mathrm{H}_{2} \mathrm{O}_{2}$ due to inhibited enzymatic dismutation of superoxide anions. Thus, inhibition of SOD not only causes reactivation of intercellular apoptosis-inducing signaling but also causes a drastic decrease in the concentration of proliferationstimulating $\mathrm{H}_{2} \mathrm{O}_{2}$. In the case of direct inhibition of catalase, $\mathrm{H}_{2} \mathrm{O}_{2}$ plays an ambivalent role, as substrate for $\mathrm{HOCl}$ synthesis but also as stimulus of proliferation of surviving tumor cells.

It will be necessary to optimize this approach by defining the best mode and schedule for application of the single-domain antibodies and also to develop methods that prevent the rapid excretion of single-domain antibodies from the body.

Future applications of single-domain antibody-based tumor therapy may utilize the synergistic effects between

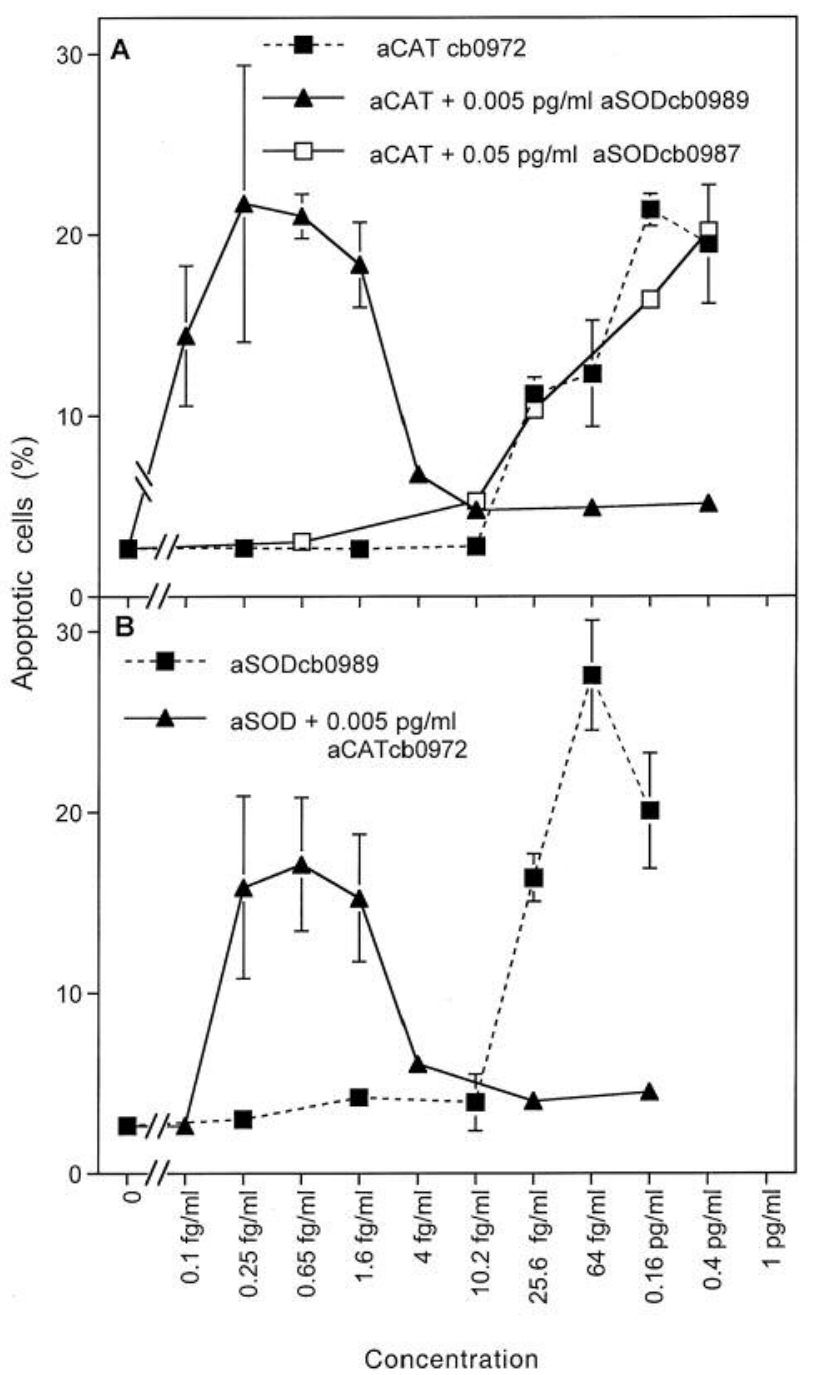

Figure 4. Synergistic effects between single-domain antibodies directed towards catalase and superoxide dismutase (SOD). At $0.005 \mathrm{pg} / \mathrm{ml}$, neutralizing aSODcb0989 had no apoptosis-inducing effect but caused a synergistic effect on apoptosis induction in MKN-45 tumor cells treated with increasing concentrations of neutralizing aCATcb0972, whereas the non-neutralizing aSODcb0987 had no synergistic effect. B: At $0.005 \mathrm{pg} / \mathrm{ml}$, neutralizing aCATcb0972 also had no apoptosisinducing effect but caused a synergistic effect on apoptosis induction in MKN-45 tumor cells treated with increasing concentrations of neutralizing aSODcb0989.

VHHs directed towards SOD and catalase, preferentially by using the hybrid molecule characterized here. The utilization of the synergistic effect between VHHs directed towards SOD or catalase with taxol, as demonstrated here, might also be very useful for application in vivo, as it should allow the concentrations of the therapeutics to be drastically lowered and thus can be predicted to reduce unwanted side-effects as well as costs. 


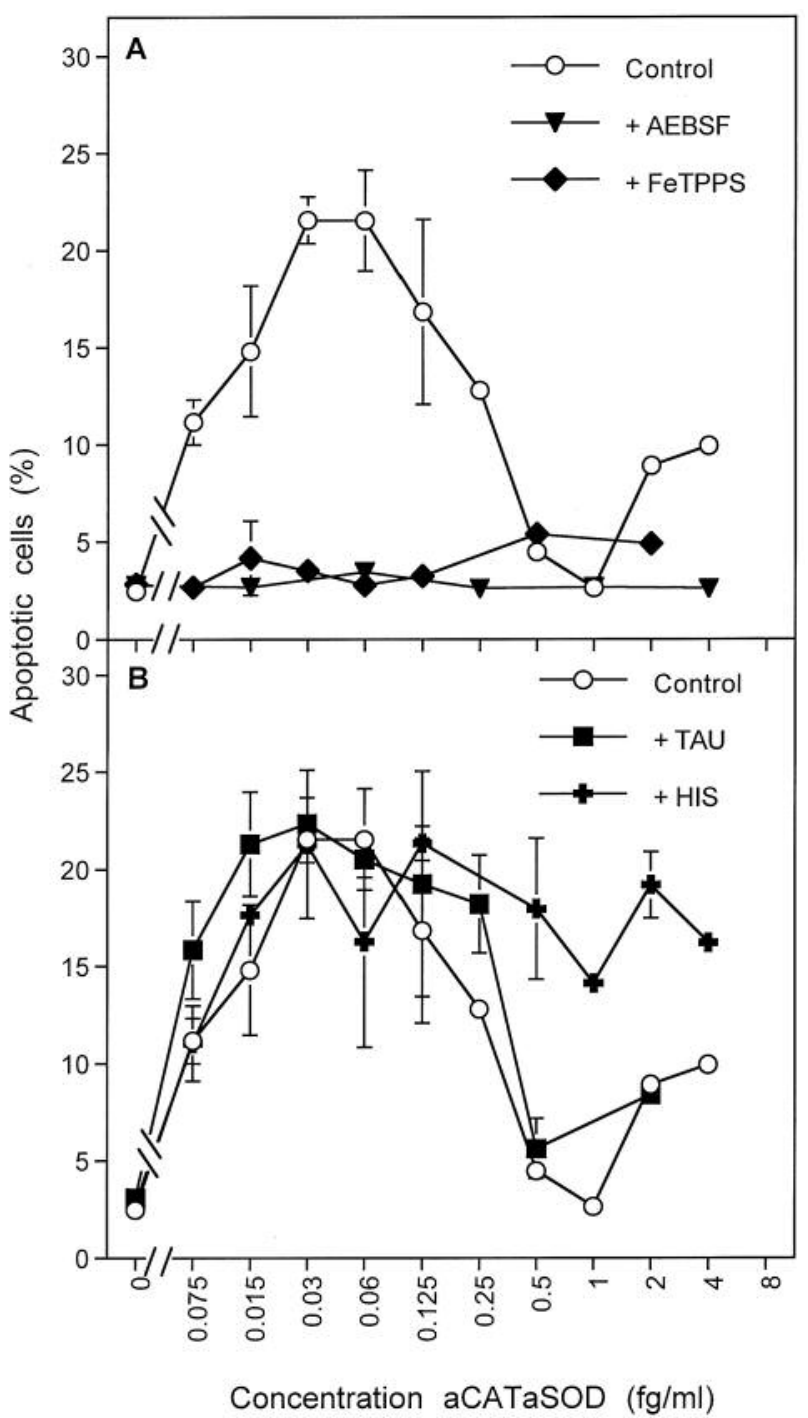

Figure 5. Apoptosis induction by a hybrid consisting of single-domain variable chain of a heavy chain-only antibody $(\mathrm{VHH})$ directed towards catalase and superoxide dismutase (aCATaSOD) is mediated by NO/peroxynitrite signaling exclusively. A: Apoptosis induction by the hybrid single-domain antibody aCATaSOD was inhibited by NADPH oxidase-1 (NOX1) inhibitor 4-(2-aminoethyl)benzenesulfonyl fluoride (AEBSF) and the peroxynitrite decomposition catalyst 5-,10-, 5-,20tetrakis(4-sulfonatophenyl)porphyrinato iron(III) chloride (FeTPPS), proving NO/peroxynitrite signaling. B: Apoptosis induction by the hybrid single-domain antibody aCATaSOD was not inhibited by the $\mathrm{HOCl}$ scavenger taurine (TAU), nor by the singlet-oxygen scavenger histidine (HIS), excluding $\mathrm{HOCl}$ signaling and the requirement of singlet oxygen for apoptosis induction.

\section{Conflicts of Interest}

The Authors have filed a patent application on the use of the singledomain antibodies described here (PCT/EP2016/052016). G.B. is sole inventor on patents owned by the University Medical Center

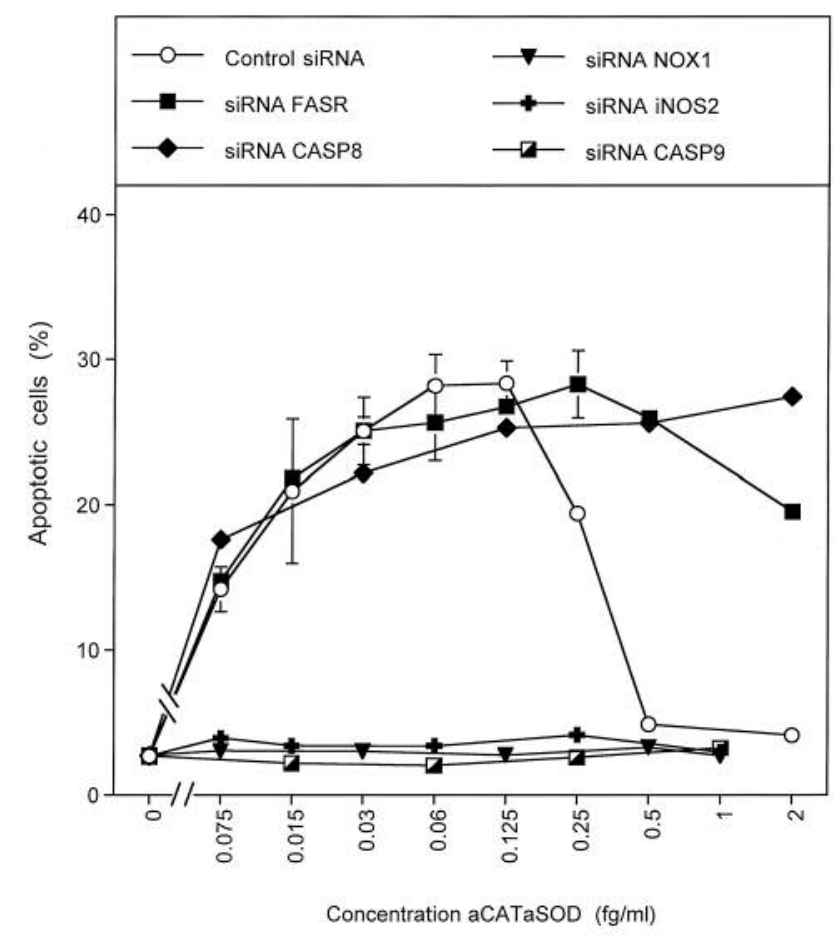

Figure 6. Small interfering ribonucleic acid (siRNA)-based analysis of reactivated intercellular reactive oxygen species/reactive nitrogen species signaling mediated by a hybrid consisting of single domain variable chain of a heavy chain-only antibodies (VHHs) directed towards catalase and superoxide dismutase (aCATaSOD) in MKN-45 cells. MKN-45 cells were treated with the indicated siRNAs for $24 \mathrm{~h}$ before increasing concentrations of hybrid aCATaSOD were added. siRNA-mediated knockdown of NOX1, inducible NO synthase-2 (iNOS2) and caspase-9 inhibited apoptosis induction by single-domain hybrid aCATaSOD, whereas knockdown of the FAS receptor (FASR), a member of the tumor necrosis factor receptor family, and caspase-8 had no inhibitory effect but did prevent the supraoptimal decline at high concentrations of the hybrid.

Freiburg, related to the synergistic effect between antibodies directed towards SOD and catalase (EP 2535352A11; US 8821875 B2) and on a patent application related to the synergistic effect between taxol and antibodies (WO2014001183A1).

\section{Acknowledgements}

The Authors thank Dr. M. Jung and her team (ChromoTek, Martinsried, Germany) for great support and professional interaction and J. Brandel (Freiburg, Germany) for valuable technical assistance for the preparation of artwork.

\section{References}

1 Irani K, Xia Y, Zweier JL, Sollott SJ, Der CJ, Fearon ER, Sundaresan $M$, Finkel $T$ and Goldschmidt-Clermont PJ: Mitogenic signalling by oxidants in Ras-transformed fibroblasts. Science 275: 1649-1652, 1997. 

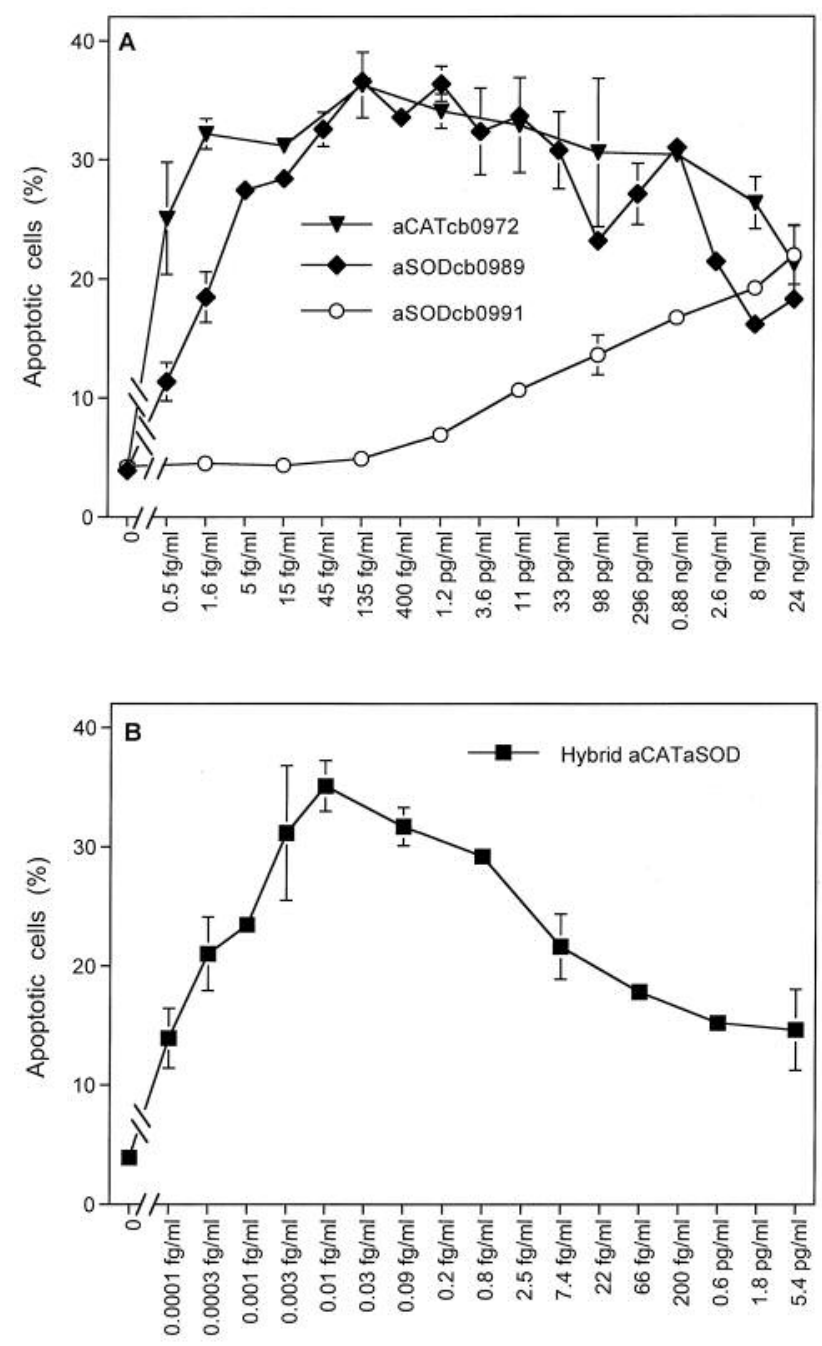

Concentration

Figure 7. A: Concentration-dependent apoptosis induction in the neuroblastoma cell line SHEP by neutralizing single-domain antibodies directed towards catalase (aCATcb0972) and SOD (aSODcb0989), and non-neutralizing SOD-binding single-domain antibodies (aSODcb0991). Note that approximately $10^{6}$-fold higher concentrations of the nonneutralizing antibodies were required to achieve the effect induced by neutralizing antibodies. B: Concentration-dependent apoptosis induction in SHEP neuroblastoma cells by a hybrid molecule consisting of neutralizing single-domains directed towards catalase and SOD.

2 Bauer G: Tumor cell protective catalase as a novel target for rational therapeutic approaches based on specific intercellular ROS signaling. Anticancer Res 32: 2599-2624, 2012.

3 Bauer G: Targeting extracellular ROS signaling of tumor cells. Anticancer Res 34: 1467-1482, 2014.

4 Bauer G: Increasing the endogenous NO level causes catalase inactivation and reactivation of intercellular apoptosis signaling specifically in tumor cells. Redox Biol 6: 353-371, 2015.

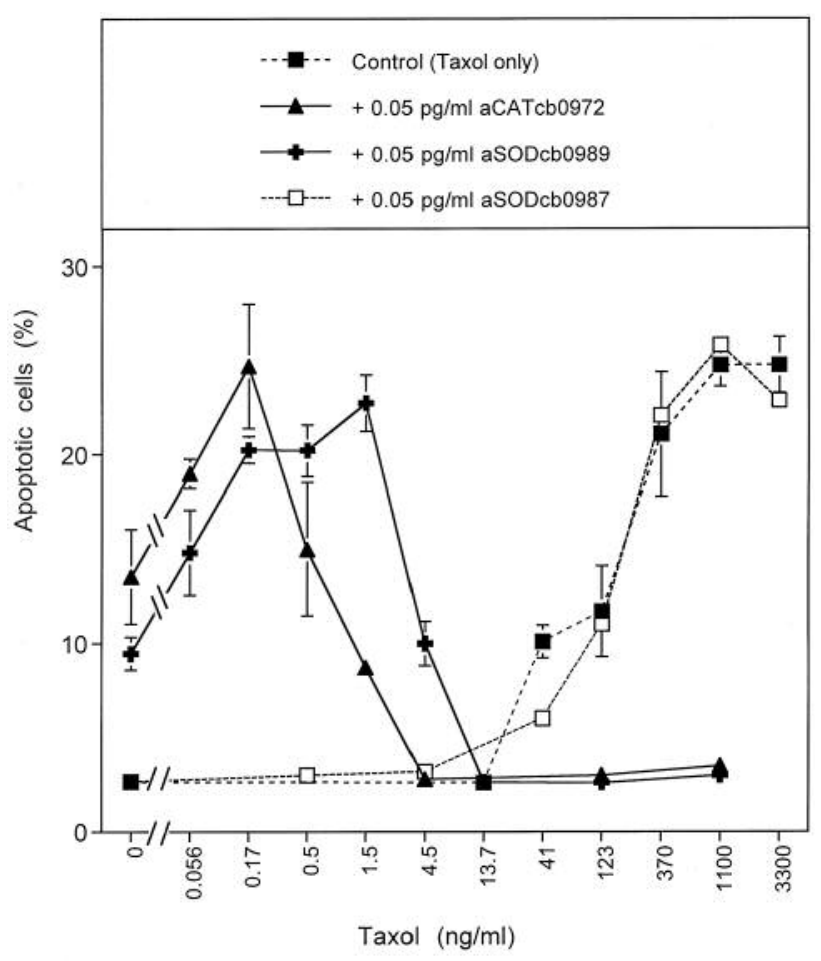

Figure 8. Synergistic effect between single-domain antibodies directed towards catalase or superoxide dismutase (SOD) with the established chemotherapeutic agent taxol. At $0.05 \mathrm{pg} / \mathrm{ml}$, neutralizing aCATcb0972 or aSODcb0989 had a valuable synergistic effect with increasing concentrations of taxol on MKN-45 human gastric carcinoma cells, whereas $0.05 \mathrm{pg} / \mathrm{ml}$ of the nonneutralizing single-domain control antibody aSODcb0987 did not.

5 Bienert GP, Schjoerring JK and Jahn TP: Membrane transport of hydrogen peroxide. Biochem Biophys Acta 1758: 994-1003, 2006.

6 Bienert GP, Møller AL, Kristiansen KA, Schulz A, Møller IM, Schjoerring JK and Jahn TP: Specific aquaporins facilitate the diffusion of hydrogen peroxide across membranes. J Biol Chem 282: 1183-1192, 2007.

7 Herdener M, Heigold S, Saran M and Bauer G: Target cellderived superoxide anions cause efficiency and selectivity of intercellular induction of apoptosis. Free Radical Biol Med 29: 1260-1271, 2000.

8 Pottgiesser S, Heinzelmann S and Bauer G: Intercellular HOClmediated apoptosis induction in malignant cells: interplay between NOX1-dependent superoxide anion generation and DUOX-related $\mathrm{HOCl}$-generating peroxidase activity. Anticancer Res 35: 5927-5943, 2015.

9 Heigold S, Sers C, Bechtel W, Ivanovas B, Schäfer R and Bauer G: Nitric oxide mediates apoptosis induction selectively in transformed fibroblasts compared to nontransformed fibroblasts. Carcinogenesis 23: 929-941, 2002.

10 Deichman G: Natural selection and early changes of phenotype of tumor cells in vivo: Acquisition of new defense mechanisms. Biochem 65: 78-94, 2000. 


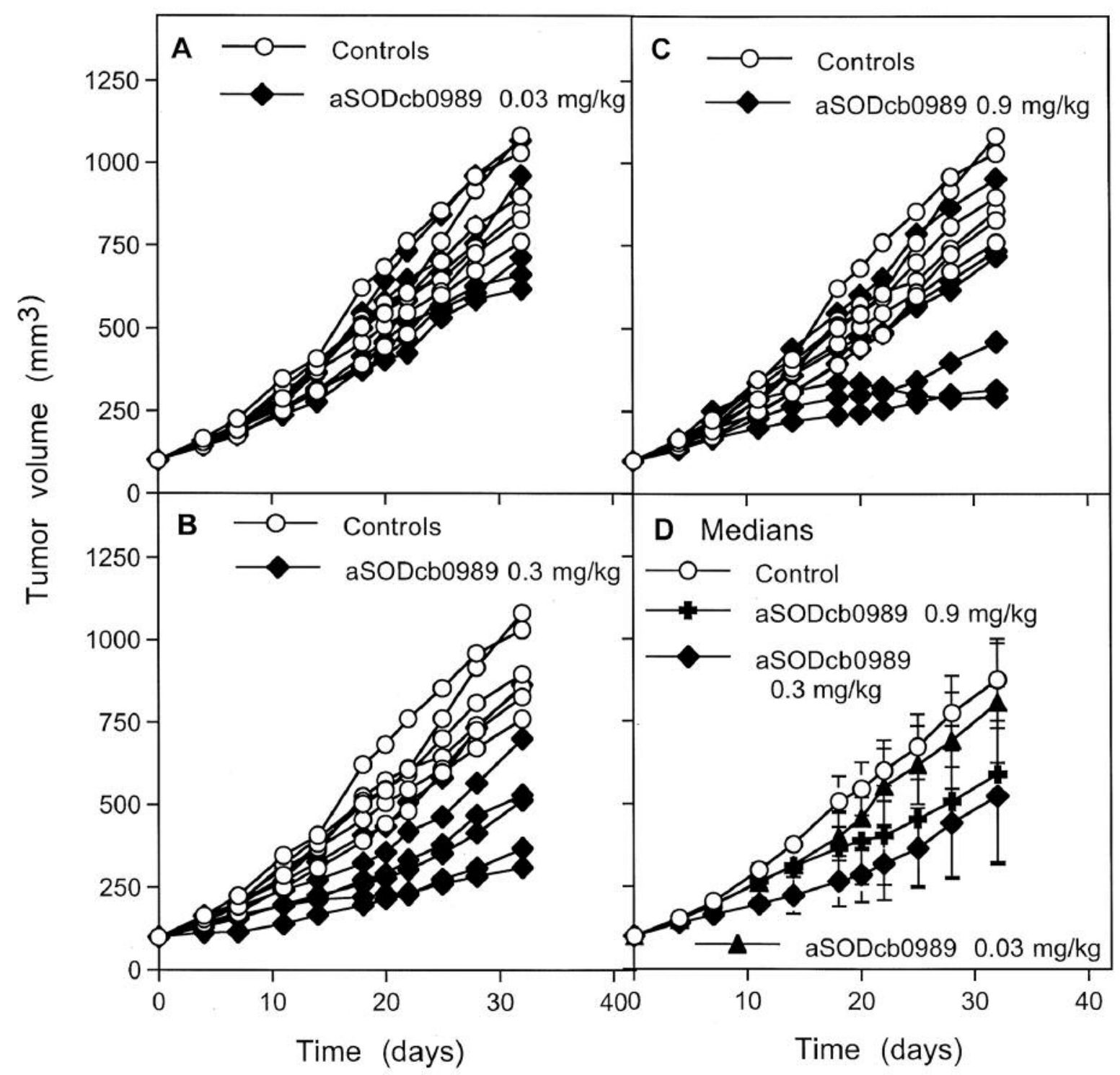

Figure 9. Kinetic study of the in vivo effect of the superoxide dismutase (SOD)-neutralizing single-domain antibody aSODcb0989 on CXF975 human colon carcinoma xenografts in immunocompromised mice. A-C: Effect of different concentrations of single-domain antibodies (closed symbol) compared to vehicle control (open symbols). D: presentation of the medians obtained from six animals per group.

11 Deichman G: Early phenotypic changes of in vitro transformed cells during in vivo progression: possible role of the host innate immunity. Sem Cancer Biol 12: 317-326, 2002.

12 Bechtel W and Bauer G: Catalase protects tumor cells against apoptosis induction by intercellular ROS signaling. Anticancer Res 29: 4541-4557, 2009.

13 Heinzelmann S and Bauer G: Multiple protective functions of catalase against intercellular apoptosis-inducing ROS signaling of human tumor cells. Biol Chem 391: 675-693, 2010.

14 Böhm B, Heinzelmann S, Motz M and Bauer G: Extracellular localization of catalase is associated with the transformed state of malignant cells. Biol Chem 396: 1339-1356, 2015.
15 Verkman AS, Hara-Chikuma $\mathrm{M}$ and Papadopoulos MC: Aquaporins - new players in cancer biology. J Mol Med 86: 523-529, 2008.

16 Bauer G. HOCl-dependent singlet oxygen and hydroxyl radical generation modulate and induce apoptosis of malignant cells. Anticancer Res 33: 3589-3602, 2013.

17 Kono Y and Fridovich I: Superoxide radical inhibits catalase. J Biol Chem 257: 5751-5754, 1982.

18 Shimizu N, Kobayashi K and Hayashi K: The reaction of superoxide radical with catalase. Mechanism of the inhibition of catalase by superoxide radical. J Biol Chem 259: 4414-4418, 1984. 
19 Fridovich I: Biological effects of the superoxide radical. Arch Biochem Biophys 247: 1-11, 1986.

20 Muyldermans S: Nanobodies: Natural single-domain antibodies. Ann Rev Biochem 82: 775-797, 2013.

21 Cortez-Retamozo V, Lauwereyi M, Hassanzadeh GHG, Gobert M, Conrath K, Muyldermans S, De Baetselier P and Revets H: Efficient tumor targeting by single-domain antibody fragments of camels. Int J Cancer 98: 456-462, 2002.

22 Temme $\mathrm{J}$ and Bauer G: Low-dose gamma irradiation enhances superoxide anion production by nonirradiated cells through TGF- $\beta 1$-dependent bystander signaling. Rad Res 179: 422-432, 2013.

23 Ivanovas B and Bauer G: Selective and nonselective apoptosis induction in transformed and nontransformed fibroblasts by exogenous reactive oxygen and nitrogen species. Anticancer Research, Anticancer Res 22: 841-856, 2002.

24 Wyllie AH, Kerr JF and Currie AR: Cell death: the significance of apoptosis. Int Rev Cytol 68: 251-274, 1980.
25 Elmore S: Apoptosis: a review of programmed cell death. Toxicol Pathol 35: 495-516, 2007.

26 Bauer G, Bereswill S, Aichele P and Glocker E: Helicobacter pylori protects oncogenically transformed cells from reactive oxygen species-mediated intercellular induction of apoptosis. Carcinogenesis 35: 1582-1591, 2014.

27 Scheit K and Bauer G: Direct and indirect inactivation of tumor cell protective catalase by salicylic acid and anthocyanidins reactivates intercellular ROS signaling and allows for synergistic effects. Carcinogenesis 36: 400-411, 2015.

Received August 30, 2016

Revised October 7, 2016

Accepted October 10, 2016 